\title{
Tonicity-responsive enhancer binding protein haplodeficiency attenuates seizure severity and NF- $\kappa$ B-mediated neuroinflammation in kainic acid-induced seizures
}

\author{
HJ Shin ${ }^{1,3}$, H Kim ${ }^{1,3}$, RW Heo ${ }^{1}$, HJ Kim ${ }^{1}$, WS Choi ${ }^{1}$, HM Kwon ${ }^{2}$ and GS Roh ${ }^{\star, 1}$
}

Kainic acid (KA)-induced seizures followed by neuronal death are associated with neuroinflammation and blood-brain barrier (BBB) leakage. Tonicity-responsive enhancer binding protein (TonEBP) is known as a transcriptional factor activating osmoprotective genes, and in brain, it is expressed in neuronal nuclei. Thus dysregulation of TonEBP may be involved in the pathology of KA-induced seizures. Here we used TonEBP heterozygote $(+I-)$ mice to study the roles of TonEBP. Electroencephalographic study showed that TonEBP $(+I-)$ mice reduced seizure frequency and severity compared with wild type during KA-induced status epilepticus. Immunohistochemistry and western blotting analysis showed that KA-induced neuroinflammation and BBB leakage were dramatically reduced in TonEBP $(+I-)$ mice. Similarly, TonEBP-specific siRNA reduced glutamate-induced death in HT22 hippocampal neuronal cells. TonEBP haplodeficiency prevented KA-induced nuclear translocation of NF- $\kappa \mathrm{B}$ p65 and attenuated inflammation. Our findings identify TonEBP as a critical regulator of neuroinflammation and BBB leakage in KA-induced seizures, which suggests TonEBP as a good therapeutic target.

Cell Death and Differentiation (2014) 21, 1095-1106; doi:10.1038/cdd.2014.29; published online 7 March 2014

Seizure-induced neuronal cell death is mainly associated with neuroinflammation and blood-brain barrier (BBB) breakdown. ${ }^{1-3}$ Reactive gliosis and inflammatory cytokines contribute to neuronal excitability and seizure severity, leading to neuronal cell death. ${ }^{4,5}$ In addition to inflammatory responses, BBB leakage results in detrimental seizure phenotype by causing brain edema formation. ${ }^{6,7}$ Recent papers showed that the brain edema induced by BBB leakage in seizures is associated with nuclear factor $-\kappa \mathrm{B}(\mathrm{NF}-\kappa \mathrm{B})$ activation and ionic imbalance. ${ }^{8,9}$ Thus, controlling the severity of neuroinflammation and BBB leakage may be critical to protect against seizure-induced neuronal death.

Tonicity-responsive enhancer binding protein (TonEBP) is well known in the kidney as a transcriptional factor of the Rel family that includes NF- $\kappa$ B and calcineurin-independent NF of activated T-cell (NFAT5) proteins. ${ }^{10}$ TonEBP in response to hypertonicity acts as a transcriptional activator to induce osmoprotective genes. ${ }^{11}$ TonEBP was shown to translocate into the nucleus and induce cyclooxygenase 2 (COX-2) enzyme in renal epithelial cells under hypertonic conditions. ${ }^{12}$ Not limited in the kidney, TonEBP is expressed in various tissues, including brain, heart, and thymus. ${ }^{11,13} \mathrm{~A}$ recent report showed that TonEBP regulates the expression of Tolllike receptor-induced genes in macrophages depending on pathogenic intensity. ${ }^{14}$ Particularly in the brain, TonEBP is predominantly expressed in the nuclei of neurons, ${ }^{15,16}$ and neurons are more susceptible in seizure-induced death compared with glia. However, inflammatory roles of neuronal TonEBP on pathology of seizures are not known yet.

Brain is enclosed by a skull, and tissues inside are necessary to have adaptive mechanisms to osmolality changes similar to those in kidney. ${ }^{16,17}$ Although the physiological roles of TonEBP have been investigated in the rat brain following systemic hypertonicity, ${ }^{15,16}$ not many studies are done considering TonEBP as a transcriptional factor of modulating pathology of neurological diseases. Recently, it has been reported that TonEBP is necessary for transcriptional regulation of aquaporin 4 (AQP4) for astrocytic swelling in kainic acid (KA)-treated hippocampus, and TonEBP-targeted silencing reduced AQP4 expression in primary astrocytes. ${ }^{18}$ However, the effect of TonEBP haploinsufficiency in vivo following KA systemic injection has not been fully studied.

\footnotetext{
${ }^{1}$ Department of Anatomy and Neurobiology, Institute of Health Sciences, Medical Research Center for Neural Dysfunction, Gyeongsang National University School of Medicine, Gyeongnam, Republic of Korea and ${ }^{2}$ School of Nano-Biotechnology and Chemical Engineering, Ulsan National Institute of Science and Technology, Ulsan, Republic of Korea

*Corresponding author: GS Roh, Department of Anatomy and Neurobiology, Institute of Health Sciences, Medical Research Center for Neural Dysfunction, Gyeongsang National University School of Medicine, 15, Jinju-daero 816 Beon-gil Jinju, Gyeongnam 660-751, Republic of Korea. Tel: +82 55 772 8035 ; Fax: +82 55 772 8039 ; E-mail: anaroh@gnu.ac.kr

${ }^{3}$ These authors equally contributed to this work.

Abbreviations: KA, kainic acid; BBB, blood-brain barrier; TonEBP, tonicity-responsive enhancer-binding protein; NF- $\kappa b$, nuclear factor- $\kappa$ B; COX-2, cyclooxygenase 2; AQP4, aquaporin 4; APD, ammonium pyrrolidine dithiocarbamate; HMGB1, high mobility group box-1; RAGE, receptor for advanced glycation end products; GFAP, glial fibrillary acidic protein; VEGF, vascular endothelial growth factor; VCAM-1, vascular cell adhesion molecule-1

Received 11.11.13; revised 26.1.14; accepted 30.1.14; Edited by N Bazan; published online 07.3.14
} 
In the present study, to determine the role of TonEBP in KA-induced seizures, we used TonEBP heterozygote mice and examined the effect on neuroinflammation and BBB leakage. We identified TonEBP as a critical regulator in both neuroinflammation and BBB leakage and showed that reducing TonEBP expression protected against seizureinduced neuronal death.

\section{Results}

KA treatment increases nuclear TonEBP expression in the hippocampal neurons. Previous studies have shown that TonEBP is mainly expressed in neurons and associated with inflammation and tonicity regulation, ${ }^{15,19}$ and strategies of modulating this expression may represent novel therapies for KA-induced seizures. Therefore, we first examined the expression level and localization of TonEBP after KA treatment by immunofluorescence staining and western blotting analysis (Figure 1). We found that TonEBP is weakly expressed in the nuclei of hippocampal neurons in control mice and highly expressed in the nucleus of hippocampal neurons at $24 \mathrm{~h}$ after KA treatment. The majority of NeuN-positive neurons from KT-treated mice showed strong TonEBP nuclear staining (Figure 1a). A few glial fibrillary acidic protein (GFAP)-positive astrocytes showed TonEBP nuclear staining (Figure 1b). However, microglial marker lba-1 staining is not colocalized with any of TonEBP-positive cells (Supplementary Figure S1). This result confirmed that KA induces TonEBP expression mostly in neuronal cells, whereas only a few in glial cells.

To analyze TonEBP expression quantitatively, we performed western blotting using hippocampal total lysates and nuclear fractions from control and KA-treated mice. As shown in Figures $1 \mathrm{c}$ and d, TonEBP from total lysates was slightly decreased at $6 \mathrm{~h}$ after KA treatment; however, the expression level of TonEBP from nuclear fractions was dramatically increased in KA-treated mice.

In parallel, we examined the nuclear expression of TonEBP in vitro in HT22 hippocampal neuronal cells where cell death was induced by glutamate. First, we confirmed glutamatemediated cytotoxicity by 3-(4,5-dimethyldiazol-2-yl)-2,5diphenyltetrazolium bromide (MTT) assay (Supplementary Figure S2a). Then the expression level of TonEBP was examined in control and glutamate-treated HT22 cells (Supplementary Figures S2b and c). Although whole cellular TonEBP level was decreased in glutamate-treated HT22 cells, the nuclear level was increased. Taken together, these results suggest that nuclear TonEBP expression has a critical role in neuronal toxicity in KA-treated mice in vivo and glutamate-treated HT22 cells in vitro.

Nuclear TonEBP is associated with KA-induced NF- $\kappa$ B nuclear translocation. NF- $\kappa \mathrm{B}$ p65 phosphorylation (Ser 536) is known to be related to neuronal excitotoxicity and vulnerability to seizures, ${ }^{20}$ and we confirmed this in our KAtreated seizure mice (Supplementary Figure S3). The phosphorylation and nuclear translocation of $\mathrm{NF}-\kappa \mathrm{B}$ were significantly increased after KA treatment (Supplementary Figures $S 3 a$ and b). APD, an NF- $\kappa$ B inhibitor, inhibited the $\mathrm{NF}-\kappa \mathrm{B}$ nuclear translocation and attenuated $\mathrm{KA}$-induced seizure activity (Supplementary Figures S3b and c). This confirmed that KA-mediated excitotoxicity is associated with $\mathrm{NF}-\kappa \mathrm{B}$ signaling pathway. Thus we examined whether the nuclear TonEBP expression correlates with KA-induced $N F-\kappa B$ phosphorylation and neuronal death. We performed terminal deoxynucleotidyl transferase dUTP nick end labeling (TUNEL) and immunofluorescence staining using anti-p-NF- $\kappa$ B p65 (Ser 536) antibody (Figure 2). There was a dramatic increase in TUNEL-positive cells after KA treatment; however, TonEBP expression was not colocalized with these apoptotic cells (Figure 2a). As expected, NF- $\kappa$ B p65 (Ser 536) phosphorylation was completely overlapped in TonEBP-positive cells (Figure $2 b$ ). These data suggest that nuclear expression of TonEBP may activate $N F-\kappa B$ signaling in KA-induced early inflammatory stages before apoptotic cell death occurs. In addition, we also confirmed the dramatic increase in nuclear expression levels of TonEBP and NF- $\kappa$ B in glutamate-treated HT22 cells (Supplementary Figure S2d).

TonEBP heterozygote mice show anticonvulsant effects on KA-induced seizures. We next examined whether reducing the expression level of TonEBP affects KA-induced seizure severity in vivo. We obtained TonEBP $(+/-)$ mice and evaluated seizure behaviors compared with WT mice. First, we investigated the properties of status epilepticus induced by KA treatment by monitoring electroencephalographic (EEG) recordings. A characteristic pattern of spontaneous seizures was displayed in WT and TonEBP $(+/-)$ mice after KA treatment (Figure 3a). The frequency of these seizure spikes was dramatically reduced in KAtreated TonEBP $(+/-)$ mice compared with KA-treated WT mice. The quantitative EEG analysis showed that the duration of seizure spikes over total recording time (\%) in KA-treated TonEBP $(+/-)$ mice was five-fold decreased compared with KA-treated WT mice (Figure 3b). Consistently, TonEBP $(+/-)$ mice showed a dramatic reduction in behavioral seizure behaviors compared with WT mice (Figure 3c, Supplementary Movies 1 and 2). Then we showed that KA-induced animal mortality was attenuated, and survival rates were increased in TonEBP $(+/-)$ mice compared KA-treated WT mice (Figure 3d). Cresyl violetstained sections of $\mathrm{CA} 3$ regions were also examined in TonEBP $(+/-)$ mice where typical pyknotic nuclei found in apoptotic cells were significantly reduced (Figure $3 e$ ). These results indicate that TonEBP haplodeficiency reduces KAinduced seizure activity and neuronal death.

KA-induced neuroinflammation is reduced in the hippocampus of TonEBP heterozygote mice. Previous studies have shown that KA treatment induces neuroinflammation and microglial activation through high mobility group box-1 (HMGB1) and its receptors for advanced glycation end products (RAGE). ${ }^{21,22}$ We confirmed that hippocampal HMGB1 and RAGE expressions were increased at $2 \mathrm{~h}$ after $\mathrm{KA}$ treatment, and Iba-1 expression, which is upregulated upon microglial activation, was increased at $6 \mathrm{~h}$ after KA treatment (Supplementary Figures S4a and b). The Iba-1 immunoreactivity was intense in dentate gyrus and CA3 regions of KAtreated mice (Supplementary Figure S4c) and was dramatically decreased in TonEBP $(+/-)$ mice (Figure 4). 
a
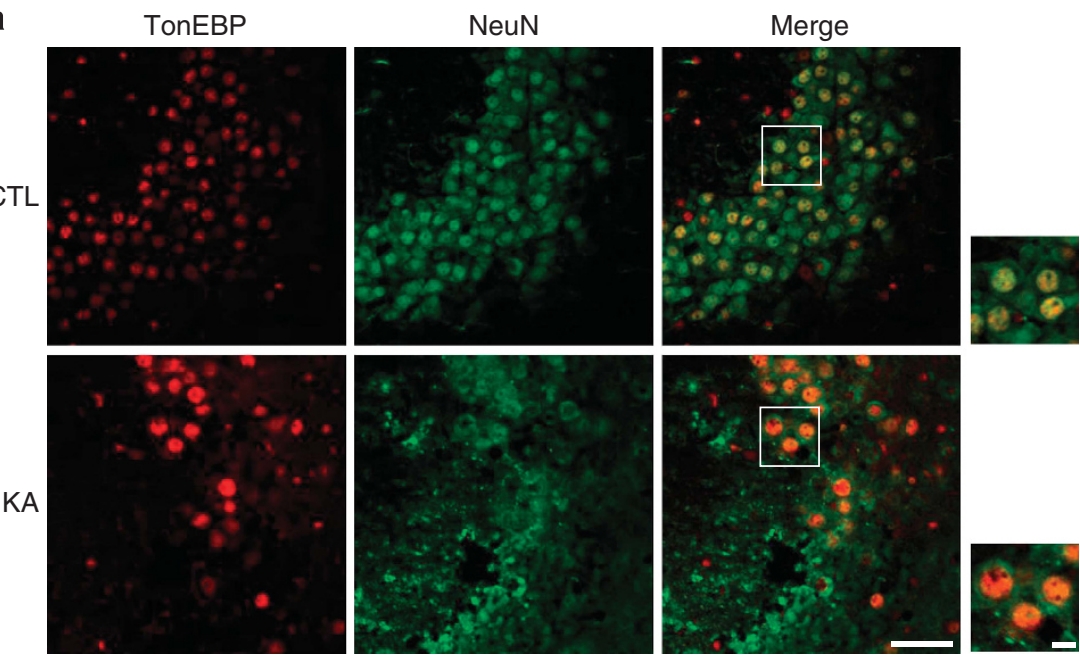

b

TonEBP
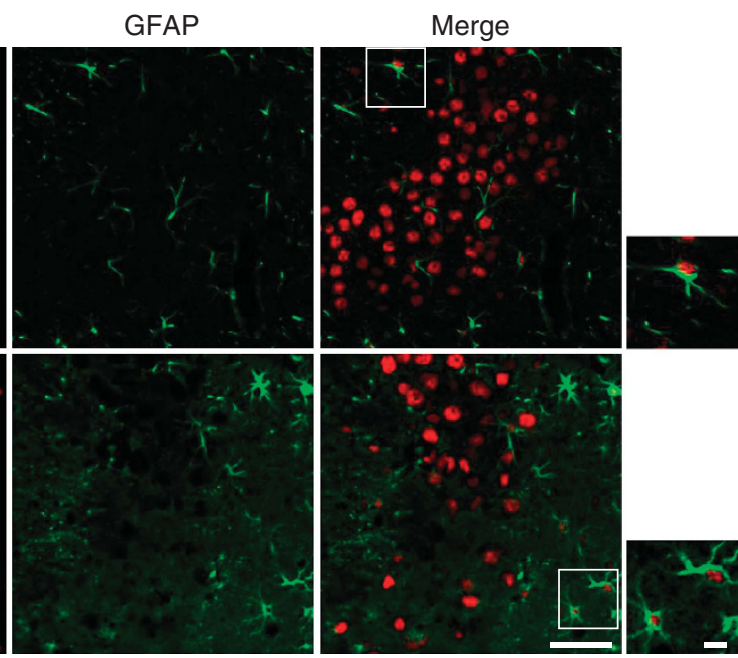

c
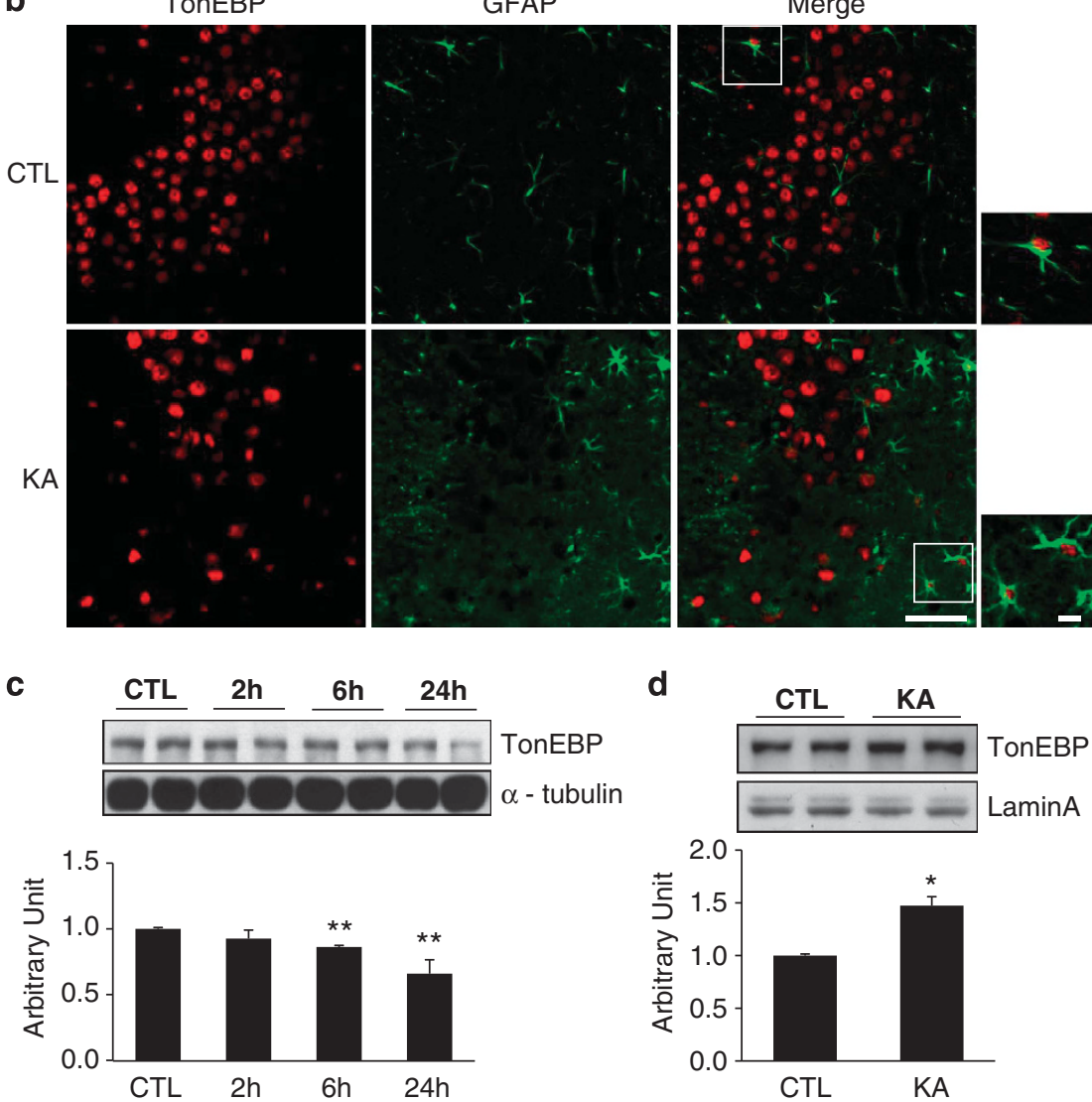

Figure 1 TonEBP expression in KA-treated hippocampal cells. (a) Representative images showing immunofluorescence of TonEBP (red) and NeuN (green) in the CA3 region of the hippocampus at $24 \mathrm{~h}$ after KA treatment. (b) Representative images of TonEBP (red) and GFAP (green). (c and d) Western blotting analysis of TonEBP in cellular lysates and nuclear fractions extracted from the hippocampus at $24 \mathrm{~h}$ after KA treatment. Quantitative comparison of protein expression was represented as arbitrary unit. Densitometry values were normalized to $\alpha$-tubulin or Lamin A. Data are shown as mean \pm S.E.M. ${ }^{*} P<0.05,{ }^{* \star} P<0.001$ versus control (CTL, 0 h)

Before we examine a neuroinflammatory response in TonEBP $(+/-)$ mice, we confirmed the reduced TonEBP expression in TonEBP $(+/-)$ mice by western blotting analysis and immunohistochemistry (Figures $5 \mathrm{a}$ and $\mathrm{b}$ ). Then we demonstrated that nuclear protein levels of TonEBP and NF$\kappa \mathrm{B}$ p65 were decreased in TonEBP $(+/-)$ mice compared with WT mice after KA treatment (Figures $5 c$ and d). The nuclear/ cytosolic ratio of TonEBP or NF- $\kappa \mathrm{B}$ p65 in TonEBP $(+/-)$ mice (0.74 or 1.22) was also decreased compared with that of WT mice (1.22 or 2.33) (Supplementary Figure S4d). Consistently, double immunofluorescence staining showed that KA-induced NF- $\kappa$ B p65 (Ser536) phosphorylation was not observed in TonEBP $(+/-)$ hippocampal neurons where TonEBP nuclear expression was also weak (Figure 5e). 
a

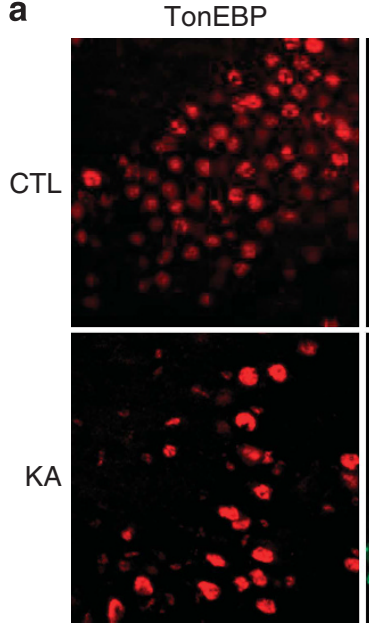

b
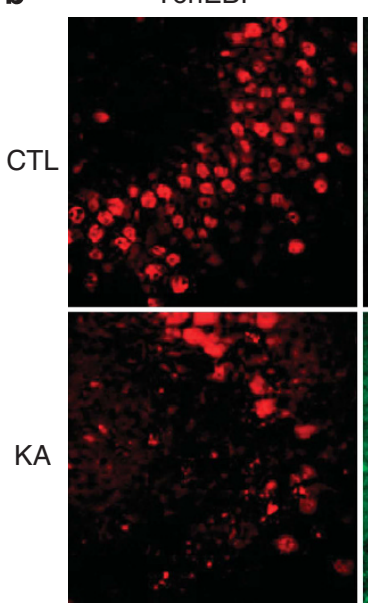

TUNEL

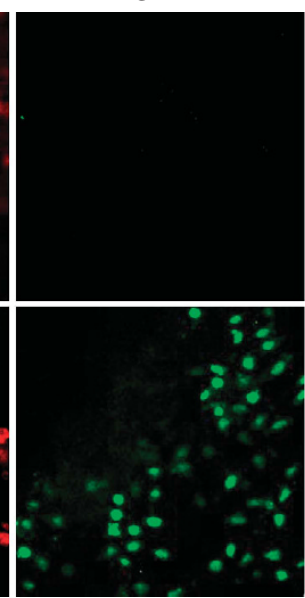

p-NFkBp65 Ser536

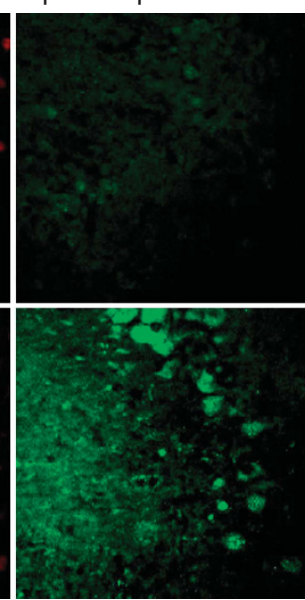

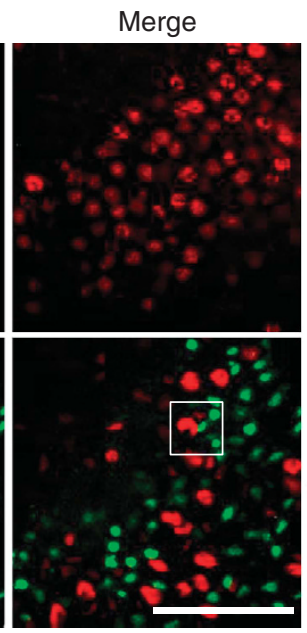
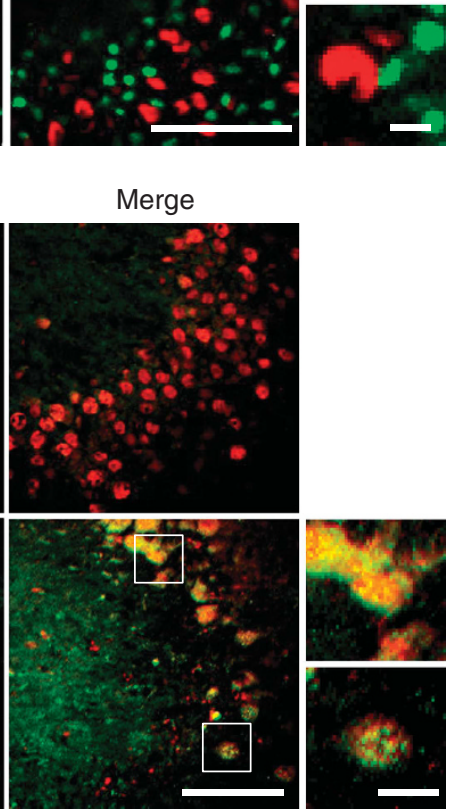

Figure 2 KA treatment induced nuclear localization of TonEBP in the hippocampus. (a) Representative images showing immunofluorescence of TonEBP (red) and TUNEL (green) in the CA3 region of the hippocampus at $24 \mathrm{~h}$ after KA treatment. (b) Representative images of TonEBP (red) and p-NF- $\kappa \mathrm{B}$ p65 (green). Scale bar $=50 \mu \mathrm{m}$ (inset, $10 \mu \mathrm{m}$ )

Next, we examined hippocampal COX-2 expression in $\operatorname{TonEBP}(+/-)$ mice. Preliminarily, we confirmed that COX-2 expression was significantly increased at $6 \mathrm{~h}$ after KA treatment (Supplementary Figure S4e); however, in TonEBP $(+/-)$ mice, it was dramatically reduced (Figure 6a). In double immunofluorescence staining, intensive COX-2stained cells induced by KA were not observed in TonEBP $(+/-)$ mice (Figure 6b). These data suggest that KA-induced nuclear TonEBP expression activates NF- $\kappa$ B mediated COX2 inflammatory signaling, and in TonEBP $(+/-)$ mice, overall inflammatory responses have been significantly reduced.

In parallel, the neuroprotective effect by reducing TonEBP expression was examined in glutamate-treated HT22 cells in vitro (Supplementary Figure S5). HT22 cells were transfected with different concentrations of TonEBP-specific siRNA, and the silencing of TonEBP reached to about $40 \%$ of control (CTL) or scrambled siRNA (Scr) at $50 \mathrm{nM}$ where minimal cell toxicity was shown among the concentrations tested (Supplementary Figures S5a and b). Then we examined whether TonEBP silencing in a condition of TonEBP haploinsufficiency mimicking TonEBP $(+/-)$ mice protects against glutamate-induced cytotoxicity by MTT assay (Supplementary Figure S5c). The percentage of difference in cell viability was $17 \%$ higher in glutamate- and TonEBP siRNA-treated cells compared with glutamate-treated cells $(P=0.002)$. In addition, round-shaped dying cells in glutamate-treated HT22 cells were not seen when TonEBP expression was silenced (Supplementary Figure S5d). The nuclear TonEBP expression was not induced by glutamate in TonEBP siRNA-treated cells where COX-2 expression was also not significantly induced (Supplementary Figures S5e and f).

\section{Hippocampal vascular endothelial growth factor (VEGF)} and AQP4 expressions are not induced by KA treatment in TonEBP heterozygote mice. In addition to neuroinflammation, BBB leakage also induces seizures and aggravates neuronal death. ${ }^{7}$ We showed KA-induced increase in expression of several BBB leakage markers, including IgG, VEGF, and vascular cell adhesion molecule-1 (VCAM-1) (Supplementary Figures S6a-C). Expression levels of IgG and VEGF were significantly induced at $6 \mathrm{~h}$ after KA 
a

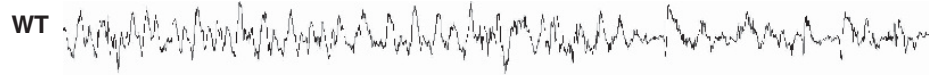

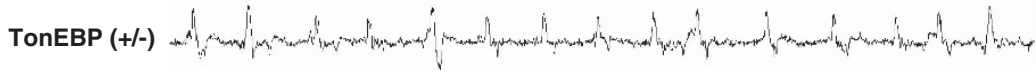
$100 \mu \mathrm{VL}$ 1s

b

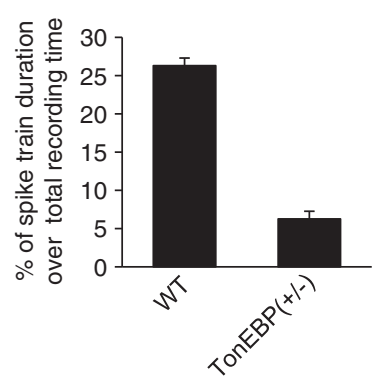

d

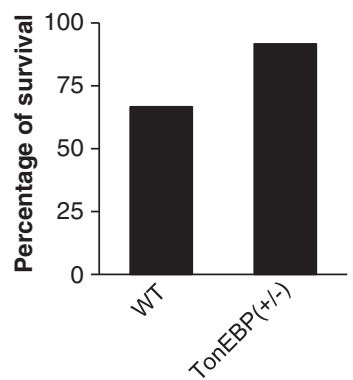

C

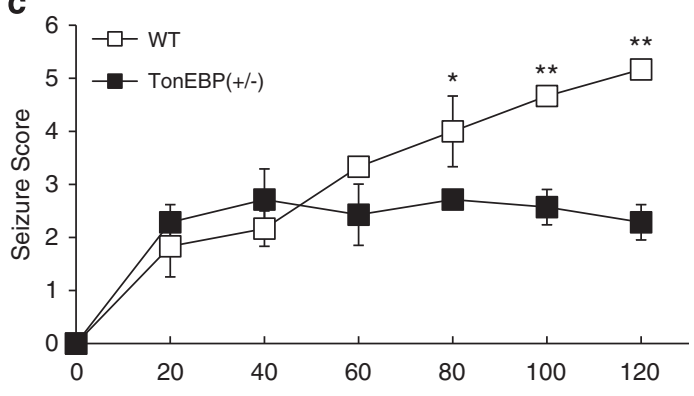

e

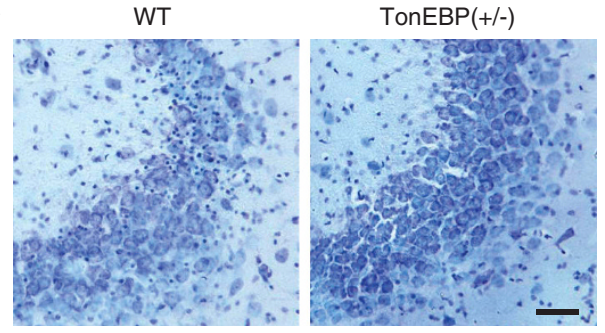

Figure 3 TonEBP $(+/-)$ mice reduced seizure activity and hippocampal cell death following KA-induced seizures. (a) EEG recording of a spontaneous seizure in a wildtype (WT) mouse and a TonEBP $(+/-)$ mouse. (b) Quantitation of EEG recordings was made at $2 \mathrm{~h}$ after KA injection in WT and TonEBP $(+l-)$ mice $(n=3$ per group). (c) The behavioral seizure scores in WT and TonEBP $(+/-)$ mice. Scores were tabulated every 20 min (WT; $n=6$, TonEBP $(+l-)$; $n=7)$. (d) Percentage of animal survival for $24 \mathrm{~h}$ after KA injection in WT and TonEBP $(+/-)$ mice (WT; $n=15$, TonEBP $(+l-), n=12)$. (e) Cresyl violet-stained sections from KA-treated WT and TonEBP $(+I-)$ mice. In KA-treated WT mice, significant neuronal death appeared as pyknotic nuclei. Scale bar $=50 \mu \mathrm{m}$. Data are shown as mean \pm S.E.M. ${ }^{*} P<0.05$, ${ }^{* *} P<0.001$ versus WT

treatment, and VCAM-1 expression was induced at $24 \mathrm{~h}$ after $\mathrm{KA}$ treatment. We also confirmed VCAM-1 expression in astrocytes where BBB leakage occurs in KA-treated hippocampus (Supplementary Figure S6d). AQP4, expressed in astrocytes, is known to control water transport, and altered water homeostasis in the brain is associated with seizure susceptibility. ${ }^{23,24}$ We first showed that AQP4 expression was markedly increased at $24 \mathrm{~h}$ after KA treatment (Supplementary Figure S6e). Brain edema induced by KA was examined by measuring the specific gravity of hippocampus. Specific gravity of KA-treated hippocampus was lower than that of control $(1.0513 \pm 0.0002$ versus $1.0487 \pm 0.0009, P=0.041)$. The water content was $5.34 \%$ higher in KA-treated hippocampus compared with control. In addition, we examined the effect of KA treatment on hippocampal specific gravity and water content in $\operatorname{Ton} \operatorname{EBP}(+/-)$ mice (Supplementary Figure S6f). The specific gravity of KA-treated hippocampus from WT mice was lower than that of control hippocampus. However, TonEBP $(+/-)$ mice showed much less difference in the specific gravity of KA-treated hippocampus compared with that of control. The water content in $\operatorname{TonEBP}(+/-)$ mice was $2.30 \%$ higher after $\mathrm{KA}$ treatment; however, the water content in WT was $4.54 \%$ higher after KA treatment compared with control. Changes in the water content in $\operatorname{TonEBP}(+/-)$ mice was two times less than that in WT mice. These data indicate that the degree of BBB leakage induced by $\mathrm{KA}$ was not severe in $\operatorname{Ton} \operatorname{EBP}(+/-)$ mice compared with WT mice.

We then investigated that the degree of BBB leakage induced by KA was altered in TonEBP $(+/-)$ mice. These mice are expected to be protective based on our previous results which showed that TonEBP $(+/-)$ mice have reduced seizure activity and neuroinflammation. We found that induction of VEGF and AQP4 expression by KA treatment was not robust in TonEBP $(+/-)$ mice (Figures $7 a$ and b). Consistently, immunofluorescence study (Figure 7c) showed that AQP4 expression was reduced in astrocytes after KA treatment in TonEBP $(+/-)$ mice.

In this study, we investigated the possible roles of TonEBP in KA-induced seizures, and our data suggest that KA-induced hyperexcitability increases nuclear TonEBP expression, which initiates NF- $\kappa$ B-mediated COX-2 inflammatory signaling and aggravates BBB leakage (Figure 8). 

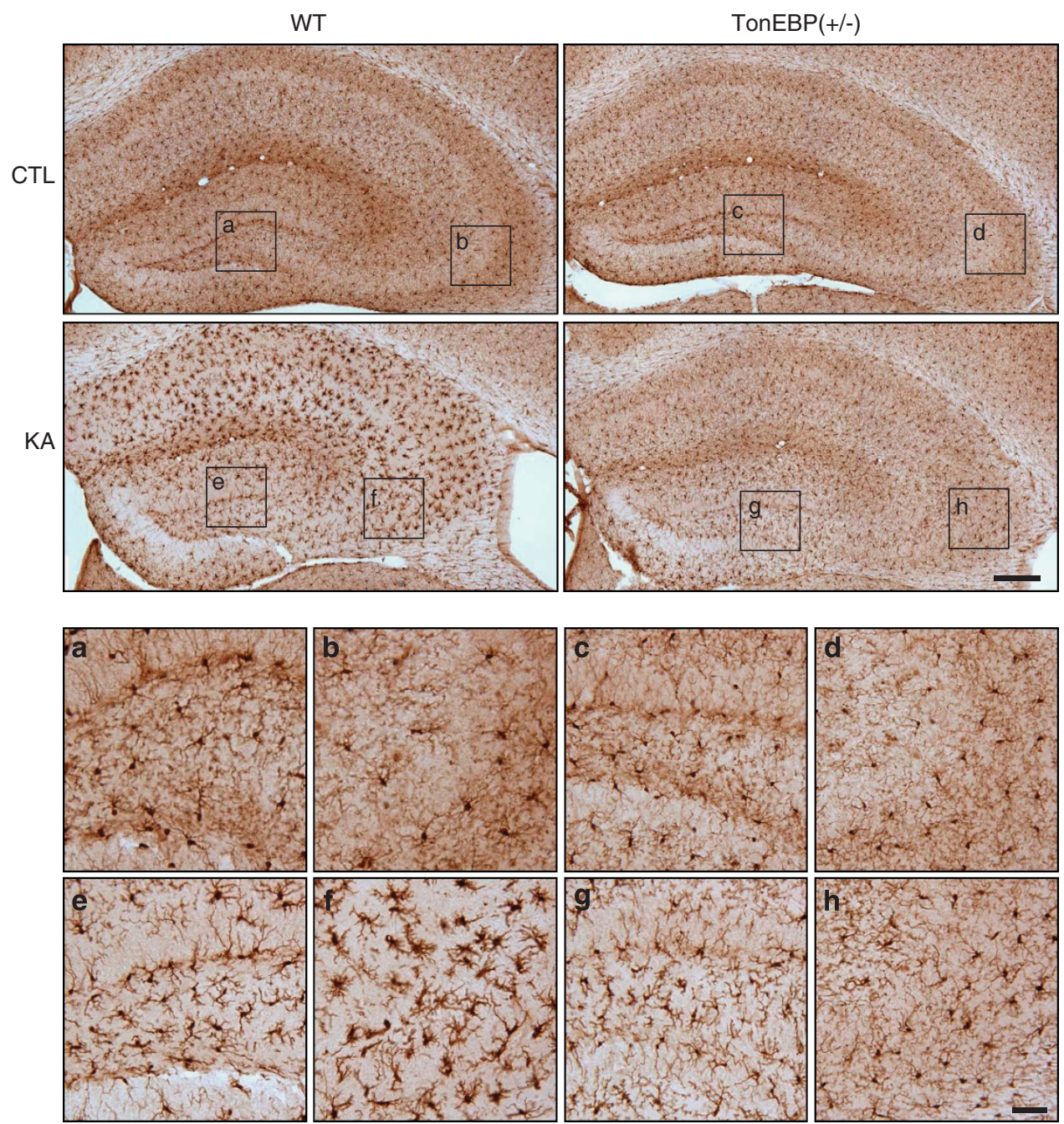

Figure 4 lba-1 immunoreactivity in the hippocampus of wild-type (WT) and TonEBP $(+/-)$ mice after KA treatment. Mice were killed at $24 \mathrm{~h}$ after KA injection. The areas in the black squares are magnified at the bottom. Scale bar $=200 \mu \mathrm{m}$ (inset, $50 \mu \mathrm{m}$ ). a, c, e, g= DG; b, d, f, h=CA3

Here we clearly showed protective effect of TonEBP haplodeficiency on the pathology of seizures and suggest reducing of TonEBP expression may be a novel therapeutic strategy for treating seizures.

\section{Discussion}

In this study, we investigated the role of a transcription factor TonEBP in KA-induced seizures. Seizure-induced neuronal cell death is associated with a complex pathology, including $\mathrm{Ca}^{2+}$ overload, hyperexcitability, oxidative stress, BBB dysfunction, and neuroinflammation. We are the first to relate TonEBP to KA-induced seizures and identify TonEBP as a critical regulator of neuroinflammation and BBB leakage. We found nuclear TonEBP elevation in response to $\mathrm{KA}$ and particularly TonEBP expression colocalized in NF- $k \mathrm{~B}$ p65 (Ser 536)-positive neurons. Treatment of a specific NF- $\kappa$ B inhibitor prevented nuclear translocation of NF- $\kappa \mathrm{B}$ p65 and reduced seizure severity. Thus, we have focused the roles of TonEBP in a NF- $\kappa$ B-mediated pathway. We demonstrated that TonEBP haploinsufficiency in vivo has protective effects against NF- $\kappa$ B-mediated neuroinflammation and BBB leakage. We showed the significant reduction in Iba-1 and COX-2 expressions as well as VEGF and AQP4 expressions in TonEBP heterozygote mice after KA injection. We conclude that TonEBP has a critical role in the pathology of KA-induced seizures, and its inhibition significantly reduces NF- $\kappa$ Bmediated cytotoxicity.

Several reports have previously demonstrated that neuronal TonEBP is expressed and upregulated following acute and prolonged systemic hypertonicity. ${ }^{15,16}$ In fact, the brain, which is enclosed by cerebrospinal fluid, is located within a skull, and hypertonicity can be induced by severe brain damage along with increased cerebral pressure and edema. Thus, TonEBP might have an important role in tonicity regulation in the brain as shown in the kidney. Numerous studies have suggested that hypertonicity-induced ionic imbalance in neurons and astrocytes leads to vasogenic edema, reactive gliosis and aggravated inflammatory responses. With this in view, we hypothesized that TonEBP regulates osmoprotective transcription and neuroinflammation in the brain and supported this hypothesis by 
a

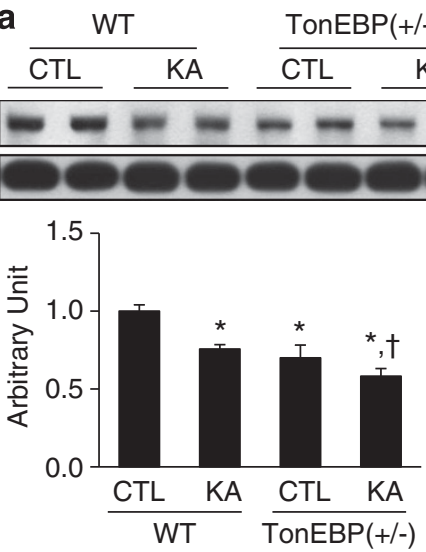

C

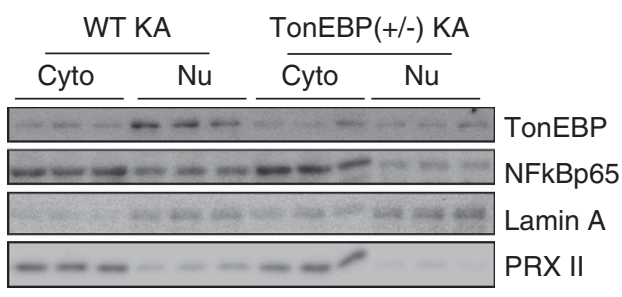

e

TonEBP $\alpha$-tubulin b

$\lessgtr$

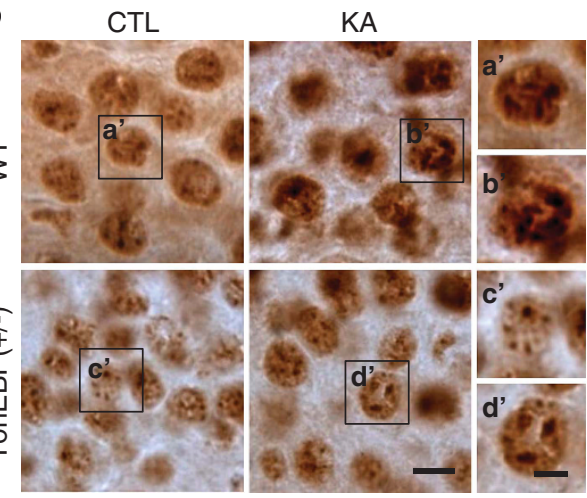

d

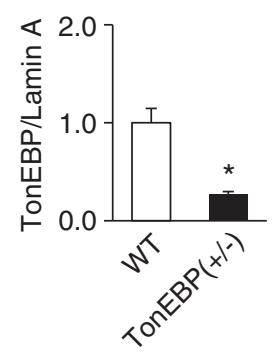

Nuclear expression

DAPI Merge

Ser536
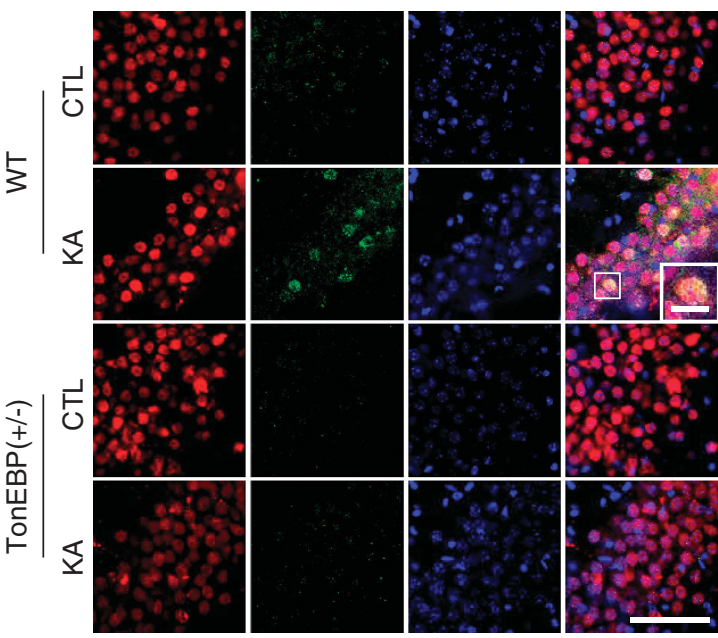

Figure 5 Nuclear expression of TonEBP and NF- $\kappa B$ after KA treatment in wild-type (WT) and TonEBP $(+/-)$ mice. Mice were killed at $24 \mathrm{~h}$ after KA injection. (a) Western blotting analysis of TonEBP. Hippocampal tissues were extracted from WT, TonEBP $(+/-)$, KA-treated WT, or KA-treated TonEBP $(+/-)$ mice. Data are shown as mean \pm S.E.M. ${ }^{*} P<0.05$ versus $C T L,{ }^{*}, \dagger<0.05$ versus KA-treated WT. (b) TonEBP immunoreactivity in the CA3 region. The boxed areas of a', b', c', and d' are magnified on the right. Scale bar $=10 \mu \mathrm{m}$ (inset, $5 \mu \mathrm{m}$ ). (c) Western blotting analysis of nuclear and cytosolic TonEBP and NF- $\kappa B$ p65 in KA-treated hippocampus of WT or TonEBP $(+/-)$ mice. (d) Nuclear TonEBP and NF- $\kappa$ B p65 in KA-treated hippocampus of WT or TonEBP $(+/-)$ mice. Data are shown as mean \pm S.E.M. ${ }^{*} P<0.05$ versus WT. (e) Representative immunofluorescence images of TonEBP (red), p-NF- $\kappa$ B p65 (green), and DAPI (4,6-diamidino-2-phenylindole; blue) in the CA3 region of the hippocampus. The area in a white square on the merged image is magnified on the right bottom. Scale bar $=50 \mu \mathrm{m}$. (inset, $10 \mu \mathrm{m}$ )

showing that TonEBP inhibition reduced KA-induced neuronal death.

We showed the elevation of nuclear TonEBP in KA-treated hippocampal neurons but not in microglia-only a few astrocytes expressed TonEBP, indicating that TonEBP regulation occurs primarily through neuronal damage responses. In addition, although TonEBP was expressed together with NF- $\kappa$ B p65 (Ser 536) and COX-2 in KA-treated hippocampal neurons, it was not colocalized with TUNEL-positive cells. We assume that KA- induced TonEBP expression triggers early inflammatory responses and does not persist in later stages of either survived or apoptotic cells. These observations are particularly interesting, given that the question of whether TonEBP has protective or detrimental role in $\mathrm{KA}$-induced neuronal death is controversial in the field. A previous study suggests that neuronal TonEBP level is upregulated to adapt to acute hypertonic changes as an osmoprotective mechanism. ${ }^{15}$ However, we found that TonEBP haploinsufficiency enhances survival in KA-induced seizures, 
a
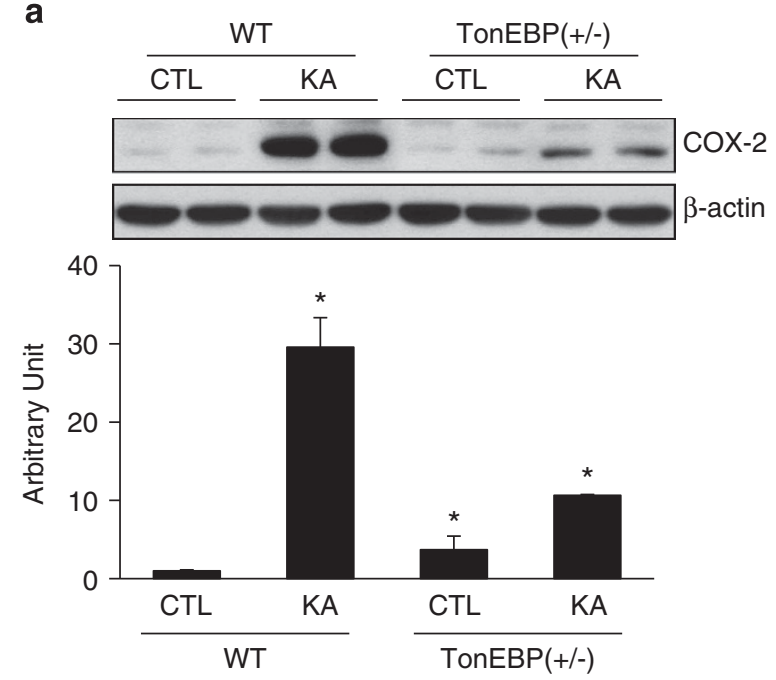

b

TonEBP

COX-2

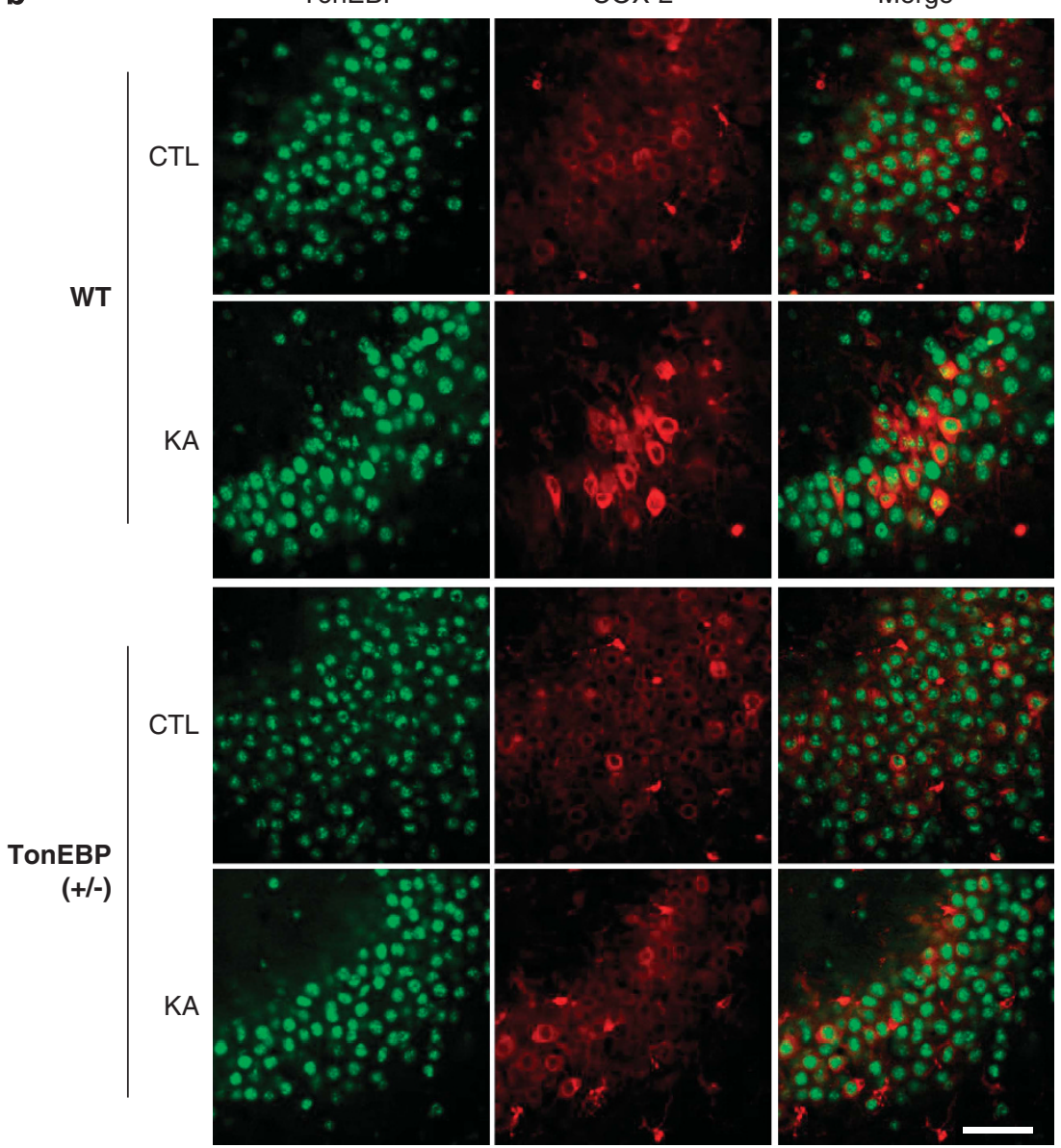

Merge

Figure 6 COX-2 expression induced by KA-treatment was reduced in TonEBP $(+/-)$ mice. (a) Western blotting analysis of COX-2. Hippocampal tissues were extracted from wild-type (WT), TonEBP $(+I-)$, KA-treated WT, or KA-treated TonEBP $(+I-)$ mice. Values were normalized to $\beta$-actin. Data are shown as mean \pm S.E.M. ${ }^{*} P<0.05$ versus WT control (CTL). (b) Representative immunofluorescence images of TonEBP (green) and COX-2 (red) in the CA3 region of the hippocampus. Scale bar $=50 \mu \mathrm{m}$

suggesting KA-induced TonEBP may be a detrimental factor eliciting chronic neuroinflammation.

Recent reports have shown that TonEBP haploinsufficiency reduced inflammatory responses and alleviated pathology of chronic diseases, including rheumatoid arthritis and atherosclerosis. $^{25,26}$ Increased TonEBP expression was observed in rheumatoid synovium, where TonEBP haploinsufficiency reduced the cytokine-induced proliferation and angiogenesis. ${ }^{26}$ TonEBP in bone marrow-derived cells was required for atherosclerotic lesion formation and macrophage 
a
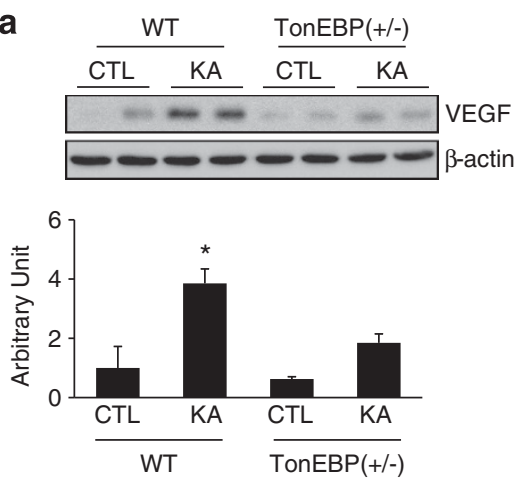

b
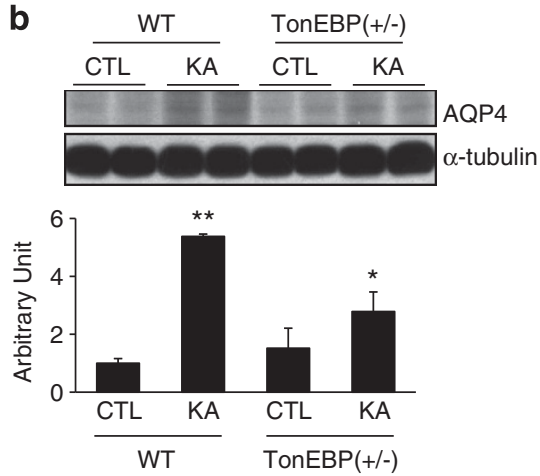

C

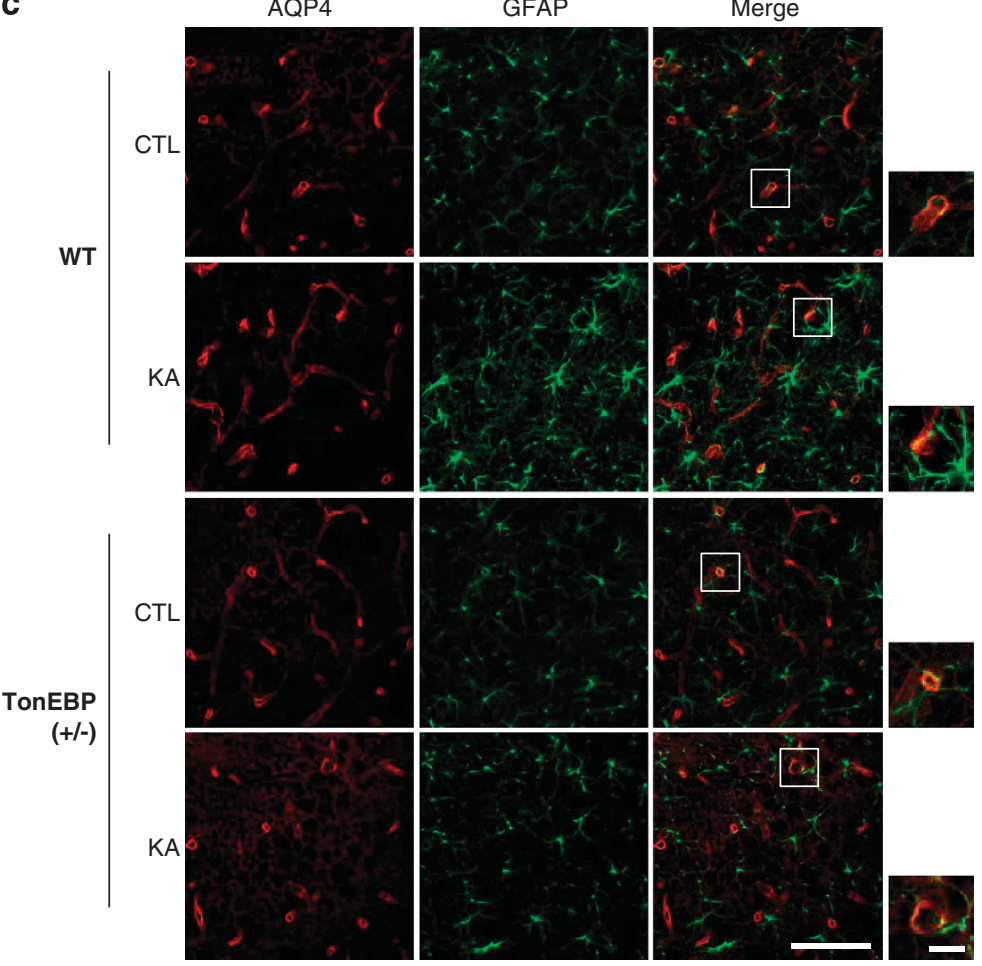

Figure 7 BBB leakage induced by KA treatment was reduced in TonEBP $(+/-)$ mice. Mice were killed at $24 \mathrm{~h}$ after KA injection. (a and $\mathbf{b})$ Western blotting analysis of VEGF and AQP4. Hippocampal tissues were extracted from wild-type (WT), TonEBP $(+/-)$, KA-treated WT, or KA-treated TonEBP $(+/-)$ mice. Values were normalized to $\alpha$-tubulin or $\beta$-actin. Data are shown as mean \pm S.E.M. ${ }^{*} P<0.05$ versus WT control (CTL), ${ }^{*} P<0.001$ versus $C$ TL. (c) Representative immunofluorescence images of AQP4 (red) and GFAP (green) in the CA3 region of the hippocampus. The areas in white squares on the merged images are magnified on the right bottom. Scale bar $=50 \mu \mathrm{m}$. (inset, $10 \mu \mathrm{m}$ )

migration. TonEBP haploinsufficiency led to a significant reduction of aortic lesions in in vivo model of atherosclerosis. ${ }^{25}$ Thus our study consistently supports that TonEBP haploinsufficiency reduces inflammation and relieves symptoms of inflammation-associated chronic human diseases.

In early inflammatory responses, the proinflammatory cytokines, including HMGB1 and TNF- $\alpha$, were released in injured neuronal and glial cells and leukocytes infiltrated into the brain parenchyma through broken BBB. ${ }^{27}$ HMGB1 is known to be released from necrotic and damaged cells ${ }^{28}$ and have cytokine-stimulating activity contributing to initial stages of inflammatory responses. ${ }^{29}$ Consistent to a previous report showing the HMGB1 release at $2 \mathrm{~h}$ after ischemic perfusion in brain, ${ }^{30}$ we showed that HMBG1 and RAGE (receptor for HMGB1) were increased in the hippocampus at $2 \mathrm{~h}$ after KA treatment. In supporting these early inflammatory responses, we showed that the reactive microglial activation marker lba-1 expression was increased at $2 \sim 6 \mathrm{~h}$ after KA-treatment. These molecules induce an NF- $\kappa \mathrm{B}-$ mediated COX-2 signaling pathway. ${ }^{27,31}$ COX-2 inhibitors were shown to protect CA3 neurons from KA-induced cell death and attenuate hippocampal prostaglandin production after seizure. ${ }^{32,33}$ COX-2 knockout mice were shown to be resistant to neuronal death after $\mathrm{KA}$ treatment. ${ }^{34}$ Along the same line of the study, we demonstrated that TonEBP heterozygote mice and TonEBP siRNA-treated HT22 cells showed reduced COX-2 expression after KA injection and glutamate treatment, respectively. Consistently, in renal epithelial cells, TonEBP siRNA was also shown to inhibit hypertonicity-induced COX-2 mRNA and protein expression. ${ }^{12}$ Taken together, TonEBP inhibition 


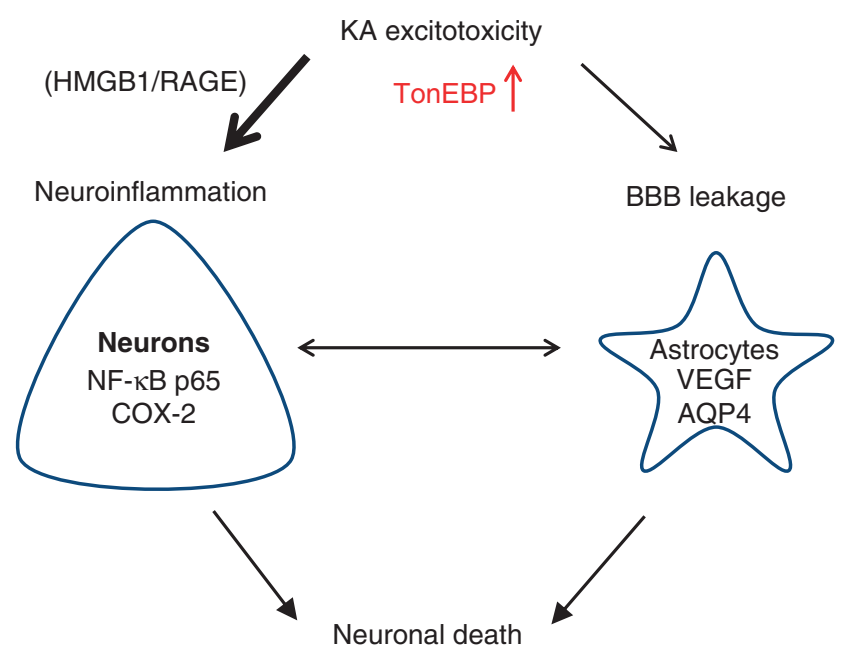

Figure 8 A schematic drawing of TonEBP-mediated pathology in KA-induced seizures. KA excitotoxicity induces neuroinflammation and BBB leakage through elevated nuclear expression of TonEBP, which activates NF- $\kappa$ B-mediated COX-2 signaling and induces BBB leakage resulting in neuronal death. We suggest that reducing TonEBP expression attenuates the pathology of KA-induced seizures

reduces COX-2 inflammatory signaling and enhances neuronal survival.

As discussed earlier, TonEBP functions as a transcription activator by binding to osmotic response element of osmoprotective genes. In addition to this classical action, TonEBP was shown to interact with $\mathrm{p} 65 \mathrm{NF}-\kappa \mathrm{B}$ subunit and enhance binding of NF- $\kappa \mathrm{B}-$ TonEBP complexes to promoters of NF- $\kappa \mathrm{B}$ responsive genes. ${ }^{19}$ With this in view, we believe that TonEBP haploinsufficiency decreased NF- $\kappa$ B-mediated transcriptional activity and attenuated the severity of neuroinflammation. Specific NF- $\kappa$ B p 65 phosphorylation and its nuclear translocation are also important in neuronal vulnerability to status epilepticus, particularly in the CA1 and CA3 pyramidal cells. ${ }^{20,35}$ NF- $\kappa$ B p65-Ser536 phosphorylation was accompanied with increased caspase- 3 activity in human fetal osteoblast cells induced by radiation. ${ }^{36}$ In the present study, we showed that p65-Ser536 NF- $k$ B phosphorylation was increased in KA-treated neurons and colocalized with TonEBP expression. However, p65-Ser536 NF- $\kappa$ B phosphorylation was not detected in KA-treated TonEBP $(+/-)$ mice. These results stress again that reducing TonEBP expression would be a good strategy to treat NF- $\kappa$ B-mediated cytotoxicity in other chronic inflammatory diseases.

In addition to neuroinflammation, BBB leakage is also associated with the progression of seizures aggravating neuroinflammation. ${ }^{7,37,38}$ Our previous study demonstrated that adiponectin pretreatment, which preserves the integrity of BBB, has neuroprotective effects in KA-induced seizures. ${ }^{1}$ Opening of the BBB by hyperosmotic treatment aggravates epileptic seizures, ${ }^{7}$ and this shows a positive correlation between BBB leakage and seizures in chronic epilepsy. In this study, we showed that TonEBP haploinsufficiency reduced BBB leakage in KA-induced seizure mice.

Emerging studies have demonstrated dysregulation of water and potassium homeostasis in patients with temporal lobe epilepsy. ${ }^{39,40} \mathrm{KA}$-induced unusual sodium ion influx and potassium ion efflux trigger neuronal hyperactivity and subsequent neuronal shrinkage. Astrocytic swelling is induced to clear excessive potassium in the extracellular space. ${ }^{41,42}$ Glial water channel AQP4 has been known to regulate water transport against osmotic gradient during neural activity, and mice lacking AQP4 are partially protected from brain edema in water intoxication and ischemic models of brain injury. ${ }^{43}$ AQP4 knockout mice showed a significantly prolonged seizure associated with a deficit in extracellular potassium clearance. ${ }^{44} \mathrm{~A}$ recent study reported that TonEBP binds to AQP4 promoter and transcriptionally activates AQP4 expression, resulting in astrocytic swelling in KA-treated mice and in ammonia-treated primary astrocytes. ${ }^{18}$ In this paper, we showed that AQP4 expression was attenuated in KA-treated TonEBP $(+/-)$ mice, where less hypertonic responses would be triggered and less inflammation would follow.

In conclusion, the present study, for the first time, demonstrates that TonEBP haploinsufficiency has a neuroprotective effect against $K A$-induced seizures by reducing $\mathrm{NF}-\kappa \mathrm{B}$-mediated inflammation and BBB leakage.

\section{Materials and Methods}

Animals. Male ICR mice (4-week old) were purchased from KOATECH Co. (Pyeongtaek, South Korea) and maintained in the animal facility at Gyeongsang National University, Gyeongnam, Republic of Korea. Male C57BL/6 wild type $(+/+)$ and TonEBP heterozygote $(+/-)$ mice (6-week old) were received from Dr. Kwon (Ulsan National Institute of Science and Technology, Ulsan, Republic of Korea). All the animal experiments were approved by the Institutional Board of Research at the Gyeongsang National University and performed in accordance with the National Institutes of Health guidelines for laboratory animal care. Mice were individually housed with an alternating $12 \mathrm{~h}$ light/dark cycle and had free access to food pellets and tap water.

\section{Drug treatment and seizure induction}

Experiment 1: Male ICR mice were treated with an intraperitoneal injection of $30 \mathrm{mg} / \mathrm{kg} \mathrm{KA}$ (Abcam, Cambridge, MA, USA) emulsified in $0.9 \%$ normal saline. Mice in the control group $(n=10)$ were injected with saline. Mice in the each experimental group ( $n=15$ per each group) were killed at 2,6 , or $24 \mathrm{~h}$ after KA treatment.

Experiment 2: TonEBP $(+/-)$ and WT mice ( $n=40$ per group) were treated with an injection of $20 \mathrm{mg} / \mathrm{kg} \mathrm{KA} \mathrm{(Abcam).} \mathrm{Mice} \mathrm{in} \mathrm{each} \mathrm{experimental} \mathrm{group} \mathrm{were}$ killed at $24 \mathrm{~h}$ after $\mathrm{KA}$ treatment.

Experiment 3: Male ICR mice were divided randomly into four groups: controls (CTL, $n=6)$, KA-induced seizure mice (KA, $n=8)$, seizure mice pretreated with ammonium pyrrolidine dithiocarbamate (APD), an NF- $\kappa B$ inhibitor (APD $+K A$, $n=8$ ), and mice treated with APD only (APD, $n=6$ ). According to a previous study, ${ }^{45}$ mice were given intraperitoneal injections of $50 \mathrm{mg} / \mathrm{kg}$ APD emulsified in $0.9 \%$ normal saline twice at $24 \mathrm{~h}$ and $30 \mathrm{~min}$ before the injection of KA. Mice were then treated with an injection of $30 \mathrm{mg} / \mathrm{kg} \mathrm{KA}$. Control mice received intraperitoneal injections of saline at the same time periods.

Monitoring after seizure induction. Mice were monitored for seizure behavior for $2 \mathrm{~h}$ after KA injection. The severity of seizures was categorized into six-point seizure scales (I-VI) as previously described. ${ }^{4}$

EEG recording. For the recording of EEG after KA-induced seizures, we used small animal radio-telemetry system from Data Sciences International (DSI, St. Paul, MN, USA). Brain electrical activity was monitored in freely mobile control or KA-treated mice. Mice were anesthetized with zoletil $(0.5 \mathrm{mg} / \mathrm{kg}$, Virbac Laboratories, Carros, France). The surgical procedure used was adapted from previous studies using similar equipment. ${ }^{46,47}$ Telemetric EEG recording was performed after surgical implantation of telemetric transmitters (TA10EA-F20; DSI). Briefly, the skin overlaying skull and neck was incised, and a subcutaneous skin pouch was created by blunt dissection underneath the skin on the back of the animal. Telemetric implants were placed into the pouch, and flexible leads were connected to 
stainless steel screws (length $2.6 \mathrm{~mm}$; thread diameter, $1 \mathrm{~mm}$ ), which were positioned epidurally over the frontal and parietal cortex $(1.5 \mathrm{~mm}$ lateral to sagittal suture and rostral to bregma) and on the contralateral side $(1.5 \mathrm{~mm}$ lateral to sagittal suture and caudal to bregma). The neck skin incision was sutured, and the remaining gap on the skull with screws and attached leads was covered with dental cement. Mice were singly housed thereafter and allowed to recover from surgery for 7 days before KA injection. EEG data were recorded with Neuro Score version 2.1 software (DSI). Offline interictal spike sorting and frequency analysis were done by Auto seizure spike train detector (DSI). To discriminate abnormal interictal spikes from physiologically occurring ones in epidural one-channel EEGs, only the spikes that were followed by a wave were manually counted. EEG was monitored for $6 \mathrm{~h}$.

Specific gravity and percentage of difference in water content. Hippocampal microdissection was performed at $24 \mathrm{~h}$ after KA treatment. Using a bromobenzene (Sigma, St. Louis, MO, USA) and kerosene (Sigma) density-gradient column, specific gravity and percentage of difference in water content were measured and calculated according to the method described by Emerson et al. ${ }^{48}$

Cell culture. HT22 mouse hippocampal cells have been used as a model system for studying glutamate-induced excitotoxicity. ${ }^{49}$ HT22 cells were maintained in Dulbecco's modified Eagle's medium (Hyclone Laboratory, Logan, UT, USA), supplemented with $10 \%$ heat-inactivated fetal bovine serum and $1 \%$ penicillin/ streptomycin (Hyclone Laboratory). HT22 cells were seeded onto 60-mm tissue culture dishes at $150000 \mathrm{cell} / \mathrm{s} / \mathrm{ml}$ in $3 \mathrm{ml}$ of growth medium for each group and grown overnight before experimental treatments. The transfection of TonEBPspecific siRNA was performed using MicroPorator (MP-100, Digital Bio Technology, Seoul, South Korea) according to the manufacturer's instructions. The sequence for TonEBP-specific siRNA is $5^{\prime}$-CCAGUUCCUACAAUGAUAACACU- $3^{\prime}$, and the sequence for scrambled siRNA is $5^{\prime}$-CCUACGCCACCAAUUUCGU- $3^{\prime}$.

Transfection efficiency was $>80 \%$ as assessed by GFP fluorescence. None of the siRNA-treated cells exhibited any significant cytotoxicity. For phase-contrast microscopy, HT22 cells were seeded on 24-well plates at 150000 cells per well. We treated cells with TonEBP-specific siRNA for $24 \mathrm{~h}$. Then $5 \mathrm{mM}$ of glutamate (Sigma) was added to each well for additional $12 \mathrm{~h}$.

Cell viability. Cell viability was assessed by measuring the metabolism of MTT. Briefly, $200 \mu \mathrm{l}$ of MTT solution $(0.5 \mathrm{mg} / \mathrm{ml})$ was added to each well of a 24-well plate, and cells were maintained for $4 \mathrm{~h}$ at $37^{\circ} \mathrm{C}$. Then $200 \mu \mathrm{l}$ of dimethyl sulfoxide was added to each well, and absorbance at $570 \mathrm{~nm}$ was measured. Cell viability was expressed as the percentage of survival compared with control, which was considered as $100 \%$ survival.

Tissue preparation and Cresyl violet staining. For tissue analysis, mice were anesthetized with zoletil $(5 \mathrm{mg} / \mathrm{kg}$, Virbac Laboratories) and then perfused transcardially with heparinized saline followed by $4 \%$ paraformaldehyde in $0.1 \mathrm{M}$ phosphate-buffered saline (PBS). After post-fixation in the same fixative for $6 \mathrm{~h}$, the brains were sequentially immersed in $0.1 \mathrm{M}$ PBS containing $15 \%$ sucrose and then in PBS containing $30 \%$ sucrose at $4{ }^{\circ} \mathrm{C}$ until they were completely submerged. The brains were cut into $40-\mu \mathrm{m}$ thick coronal sections. The sections stained with Cresyl violet were visualized with a BX51 light microscope (Olympus, Tokyo, Japan), and digital images were captured.

Protein extraction. After anesthesia with zoletil, the brains were quickly removed from the skull, and both hippocampi were dissected and frozen. Hippocampal whole cellular extracts, cytosolic fractions, nuclear fractions, and HT22 cell lysates were prepared as previously described. ${ }^{4}$ Briefly, frozen hippocampi were individually transferred to sterile $1.5-\mathrm{ml}$ microcentrifuge tubes containing $200 \mu \mathrm{l}$ of lysis buffer ( $15 \mathrm{mM}$ HEPES, pH 7.9, $0.25 \mathrm{M}$ sucrose, $60 \mathrm{mM}$ $\mathrm{KCl}, 10 \mathrm{mM} \mathrm{NaCl}, 1 \mathrm{mM}$ EGTA, $1 \mathrm{mM}$ PMSF, and $2 \mathrm{mM} \mathrm{NaF})$. Homogenized tissues or cells were incubated for $10 \mathrm{~min}$ on ice and then sonicated. Samples were then centrifuged at $4^{\circ} \mathrm{C}$ for $30 \mathrm{~min}$ at 12000 r.p.m., an the supernatants were transferred to clean vials, and samples were stored at $-80^{\circ} \mathrm{C}$

TUNEL staining. TUNEL analysis was performed to measure the degree of apoptosis in the tissues using an in situ cell death detection kit (Roche Molecular Biochemicals, Mannheim, Germany) according to the manufacturer's protocol. The stained brain sections were visualized with a confocal microscope (FV-1000, Olympus), and digital images were captured.
Double immunofluorescence. For double immunofluorescence staining, the frozen free-floating brain sections were incubated with rat anti-VCAM-1 and mouse anti-GFAP; rabbit anti-TonEBP and mouse anti-NeuN or mouse anti-GFAP; goat anti-TonEBP and rabbit anti-lba-1, rabbit anti-p-NF- $\kappa$ B p65 (Ser 536), or rabbit anti-COX-2; and rabbit anti-AQP4 and mouse anti-GFAP at $4{ }^{\circ} \mathrm{C}$ for 2 days. After washing three times with $0.1 \mathrm{M}$ PBS, sections were incubated with Alexa Fluor 488- and 594-conjugated donkey anti-rabbit, anti-mouse, or ant-goat secondary antibodies (Invitrogen, Carlsbad, CA, USA). For HT22 cell staining, cells were cultured on cover glass (MultiCell plus, CTRL Life Science, Gwangiu, South Korea) and fixed with 4\% paraformaldehyde in PBS for $10 \mathrm{~min}$ at room temperature (RT). Permeabilization was performed in PBS with $0.3 \%$ Triton $X-100$ for $10 \mathrm{~min}$ at RT. After blocking for 30 min with $5 \%$ normal donkey serum, cells were incubated with rabbit anti-TonEBP and mouse anti-NF- $\kappa B$ p 65 at $4{ }^{\circ} \mathrm{C}$ overnight. After washing with PBS, Alexa Fluor 488 and -594 secondary antibodies were added for $1 \mathrm{~h}$. Nuclei were stained with DAPI (1:10000, Invitrogen). Fluorescence was visualized with a confocal microscope (FV-1000), and digital images were captured.

Immunohistochemistry. The frozen free-floating brain sections were incubated with rabbit anti-Iba-1 or goat anti-TonEBP overnight at $4{ }^{\circ} \mathrm{C}$. After three washes with $0.1 \mathrm{M}$ PBS, sections were incubated for $1 \mathrm{~h}$ at RT with biotinylated secondary antibody. After washing three times with $0.1 \mathrm{M}$ PBS, sections were incubated in avidin-biotin-peroxidase complex solution (ABC solution; Vector Laboratories, Burlingame, CA, USA). Sections were developed with $0.025 \%$ diaminobenzidine (Sigma) containing $0.025 \% \mathrm{H}_{2} \mathrm{O}_{2}$, mounted on gelatin-coated slides, warm-dried, dehydrated through graded alcohols, cleared in xylene, and finally coverslipped with Permount (Sigma). The stained sections were visualized with a BX51 light microscope (Olympus), and digital images were captured.

Western blotting analysis. The protein concentration of each lysate was determined using a bicinchoninic acid kit (Pierce, Rockford, IL, USA), according to the manufacturer's protocol, using bovine serum albumin as a standard. Equal amounts of protein $(30 \mu \mathrm{g})$ were separated by SDS-PAGE and transferred to nitrocellulose membranes. The membranes were washed in Tris-buffered saline containing $0.5 \%$ Tween-20 and incubated with the primary antibodies mentioned in Supplementary Table S1. The samples were then incubated with their corresponding secondary antibodies. The enhanced chemiluminescence western blotting analysis system (Amersham Pharmacia Biotech, Piscataway, NJ, USA) was used for detection. To normalize the protein levels, $\alpha$-tubulin or $\beta$-actin was used as an internal control.

Statistical analysis. Differences among the time-dependent groups or among the experimental groups were determined with one-way analysis of variance, followed by post-hoc analysis with a Student-Newman-Keuls test or with a Student's t-test when only two groups were compared. Values are expressed as the mean \pm S.E.M. A $P$ value $<0.05$ was considered as statistically significant.

\section{Conflict of Interest}

The authors declare no conflict of interest.

Acknowledgements. We are grateful to Jae Yong Park for critical reading of the manuscript and helpful suggestions. We thank Chin-ok Yi for technical support in in vitro experiments. This research was supported by the Basic Science Research Program through the National Research Foundation (NRF) of Korea funded by the Ministry of Science, ICT and Future Planning (Nos. 2005-0049415 and 2011-0020163).

\section{Author contributions}

HJS and HK designed and performed most of the experiments and wrote the manuscript. RWH assisted in in vivo experiments. HJK and WSC supported the study. HMK provided reagents and discussed the interpretation of the in vivo data. GSR designed and supervised the study and wrote the manuscript. HJS and HK equally contributed to this work.

1. Jeon BT, Shin HJ, Kim JB, Kim YK, Lee DH, Kim KH et al. Adiponectin protects hippocampal neurons against kainic acid-induced excitotoxicity. Brain Res Rev 2009; 61: 81-88.

2. Saija A, Princi P, Pisani A, Santoro G, De Pasquale R, Massi M et al. Blood-brain barrier dysfunctions following systemic injection of kainic acid in the rat. Life Sci 1992; 51: 467-477. 
3. Vezzani A. Inflammation and epilepsy. Epilepsy Curr 2005; 5: 1-6.

4. Jeong EA, Jeon BT, Shin HJ, Kim N, Lee DH, Kim HJ et al. Ketogenic diet-induced peroxisome proliferator-activated receptor-gamma activation decreases neuroinflammation in the mouse hippocampus after kainic acid-induced seizures. Exp Neurol 2011; 232: 195-202.

5. Vezzani A, Friedman A, Dingledine RJ. The role of inflammation in epileptogenesis. Neuropharmacology 2013; 69: 16-24.

6. Pavlovsky L, Seiffert E, Heinemann U, Korn A, Golan H, Friedman A. Persistent BBB disruption may underlie alpha interferon-induced seizures. J Neurol 2005; 252: 42-46.

7. van Vliet EA, da Costa Araújo S, Redeker S, van Schaik R, Aronica E, Gorter JA Blood-brain barrier leakage may lead to progression of temporal lobe epilepsy. Brain 2007; 130: $521-534$

8. Kim JE, Ryu HJ, Choi SY, Kang TC. Tumor necrosis factor-alpha-mediated threonine 435 phosphorylation of p65 nuclear factor-kappaB subunit in endothelial cells induces vasogenic edema and neutrophil infiltration in the rat piriform cortex following status epilepticus. J Neuroinflammation 2012; 9: 6.

9. Shin HJ, Jeon BT, Kim J, Jeong EA, Kim MJ, Lee DH et al. Effect of the calcineurin inhibitor FK506 on $\mathrm{K}+-\mathrm{Cl}$ - cotransporter 2 expression in the mouse hippocampus after kainic acid-induced status epilepticus. J Neural Transm 2012; 119: 669-677.

10. Aramburu J, Drews-Elger K, Estrada-Gelonch A, Minguillón J, Morancho B, Santiago V et al. Regulation of the hypertonic stress response and other cellular functions by the Rel-like transcription factor NFAT5. Biochem Pharmacol 2006; 72: 1597-1604.

11. Miyakawa H, Woo SK, Dahl SC, Handler JS, Kwon HM. Tonicity-responsive enhancer binding protein, a rel-like protein that stimulates transcription in response to hypertonicity Proc Natl Acad Sci USA 1999; 96: 2538-2542.

12. Favale NO, Casali Cl, Lepera LG, Pescio LG, Fernandez-Tome MC. Hypertonic induction of COX2 expression requires TonEBP/NFAT5 in renal epithelial cells. Biochem Biophys Res Commun 2009; 381: 301-305.

13. Trama J, Lu Q, Hawley RG, Ho SN. The NFAT-related protein NFATL1 (TonEBP/NFAT5) is induced upon T cell activation in a calcineurin-dependent manner. J Immunol 2000; 165 4884-4894.

14. Buxade M, Lunazzi G, Minguillón J, Iborra S, Berga-Bolaños R, Del Val M et al. Gene expression induced by Toll-like receptors in macrophages requires the transcription factor NFAT5. J Exp Med 2012; 209: 379-393.

15. Loyher ML, Mutin M, Woo SK, Kwon HM, Tappaz ML. Transcription factor tonicityresponsive enhancer-binding protein (TonEBP) which transactivates osmoprotective genes is expressed and upregulated following acute systemic hypertonicity in neurons in brain. Neuroscience 2004; 124: 89-104.

16. Maallem S, Mutin M, Kwon HM, Tappaz ML. Differential cellular distribution of tonicityinduced expression of transcription factor TonEBP in the rat brain following prolonged systemic hypertonicity. Neuroscience 2006; 137: 51-71.

17. Law RO. Regulation of mammalian brain cell volume. J Exp Zool 1994; 268: 90-96.

18. Yi MH, Lee YS, Kang JW, Kim SJ, Oh SH, Kim YM et al. NFAT5-dependent expression of AQP4 in astrocytes. Cell Mol Neurobiol 2013; 33: 223-232.

19. Roth I, Leroy V, Kwon HM, Martin PY, Féraille E, Hasler U. Osmoprotective transcription factor NFAT5/TonEBP modulates nuclear factor-kappaB activity. Mol Biol Cell 2010; 21 3459-3474.

20. Ryu HJ, Kim JE, Yeo SI, Kim MJ, Jo SM, Kang TC. ReLA/P65-serine 536 nuclear factor-kappa B phosphorylation is related to vulnerability to status epilepticus in the rat hippocampus. Neuroscience 2011; 187: 93-102.

21. Iori V, Maroso M, Rizzi M, lyer AM, Vertemara R, Carli M et al. Receptor for advanced glycation endproducts is upregulated in temporal lobe epilepsy and contributes to experimental seizures. Neurobiol Dis 2013; 58: 102-114.

22. Muhammad S, Barakat W, Stoyanov S, Murikinati S, Yang H, Tracey KJ et al. The HMGB1 receptor RAGE mediates ischemic brain damage. J Neurosci 2008; 28: 12023-12031.

23. Nielsen S, Nagelhus EA, Amiry-Moghaddam M, Bourque C, Agre P, Ottersen OP. Specialized membrane domains for water transport in glial cells: high-resolution immunogold cytochemistry of aquaporin-4 in rat brain. J Neurosci 1997; 17: 171-180.

24. Schwartzkroin PA, Baraban SC, Hochman DW. Osmolarity, ionic flux, and changes in brain excitability. Epilepsy Res 1998; 32: 275-285.
25. Halterman JA, Kwon HM, Leitinger N, Wamhoff BR. NFAT5 expression in bone marrow-derived cells enhances atherosclerosis and drives macrophage migration. Front Physiol 2012; 3: 313.

26. Yoon HJ, You S, Yoo SA, Kim NH, Kwon HM, Yoon CH et al. NF-AT5 is a critical regulator of inflammatory arthritis. Arthritis Rheum 2011; 63: 1843-1852.

27. Rosales-Corral S, Reiter RJ, Tan DX, Ortiz GG, Lopez-Armas G. Functional aspects of redox control during neuroinflammation. Antioxid Redox Signal 2010; 13: 193-247.

28. Scaffidi $P$, Misteli T, Bianchi ME. Release of chromatin protein HMGB1 by necrotic cells triggers inflammation. Nature 2002; 418: 191-195.

29. Li J, Wang H, Mason JM, Levine J, Yu M, Ulloa L et al. Recombinant HMGB1 with cytokine-stimulating activity. J Immunol Methods 2004; 289: 211-223.

30. Qiu J, Nishimura M, Wang Y, Sims JR, Qiu S, Savitz SI et al. Early release of HMGB-1 from neurons after the onset of brain ischemia. J Cereb Blood Flow Metab 2008; 28: 927-938.

31. Bianchi R, Giambanco I, Donato R. S100B/RAGE-dependent activation of microglia via NF-kappaB and AP-1 Co-regulation of COX-2 expression by S100B, IL-1beta and TNF-alpha. Neurobiol Aging 2010; 31: 665-677.

32. Baik EJ, Kim EJ, Lee SH, Moon C. Cyclooxygenase-2 selective inhibitors aggravate kainic acid induced seizure and neuronal cell death in the hippocampus. Brain Res 1999; 843 118-129.

33. Kawaguchi K, Hickey RW, Rose ME, Zhu L, Chen J, Graham SH. Cyclooxygenase-2 expression is induced in rat brain after kainate-induced seizures and promotes neuronal death in CA3 hippocampus. Brain Res 2005; 1050: 130-137.

34. Takemiya T, Maehara M, Matsumura K, Yasuda S, Sugiura H, Yamagata K. Prostaglandin E2 produced by late induced COX-2 stimulates hippocampal neuron loss after seizure in the CA3 region. Neurosci Res 2006; 56: 103-110.

35. Perkins ND. Integrating cell-signalling pathways with NF-kappaB and IKK function. Nat Rev $\mathrm{Mol}$ Cell Biol 2007; 8: 49-62.

36. Xiao M, Inal CE, Parekh VI, Li XH, Whitnall MH. Role of NF-kappaB in hematopoietic niche function of osteoblasts after radiation injury. Exp Hematol 2009; 37: 52-64.

37. Lassmann H, Petsche U, Kitz K, Baran H, Sperk G, Seitelberger F et al. The role of brain edema in epileptic brain damage induced by systemic kainic acid injection. Neuroscience 1984; 13: 691-704.

38. Oztas B, Kaya M, Kucuk M, Tugran N. Influence of hypoosmolality on the blood-brain barrier permeability during epileptic seizures. Prog Neuropsychopharmacol Biol Psychiatry 2003; 27: 701-704.

39. Binder DK, Steinhauser C. Functional changes in astroglial cells in epilepsy. Glia 2006; 54 358-368.

40. Hsu MS, Lee DJ, Binder DK. Potential role of the glial water channel aquaporin-4 in epilepsy. Neuron Glia Biol 2007; 3: 287-297.

41. Walz W. Swelling and potassium uptake in cultured astrocytes. Can J Physiol Pharmacol 1987; 65: 1051-1057.

42. Xiao AY, Homma M, Wang XQ, Wang X, Yu SP. Role of $K(+)$ efflux in apoptosis induced by AMPA and kainate in mouse cortical neurons. Neuroscience 2001; 108: 61-67.

43. Verkman AS. Aquaporin water channels and endothelial cell function. J Anat 2002; 200: $617-627$.

44. Binder DK, Yao X, Zador Z, Sick TJ, Verkman AS, Manley GT. Increased seizure duration and slowed potassium kinetics in mice lacking aquaporin-4 water channels. Glia 2006; 53 $631-636$.

45. Santos JB, Schauwecker PE. Protection provided by cyclosporin A against excitotoxic neuronal death is genotype dependent. Epilepsia 2003; 44: 995-1002.

46. Claycomb RJ, Hewett SJ, Hewett JA. Neuromodulatory role of endogenous interleukin1 beta in acute seizures: possible contribution of cyclooxygenase-2. Neurobiol Dis 2012; 45 234-242.

47. Krestel HE, Shimshek DR, Jensen V, Nevian T, Kim J, Geng Y. A genetic switch for epilepsy in adult mice. J Neurosci 2004; 24: 10568-10578.

48. Emerson MR, Nelson SR, Samson FE, Pazdernik TL. A global hypoxia preconditioning model: neuroprotection against seizure-induced specific gravity changes (edema) and brain damage in rats. Brain Res Brain Res Protoc 1999; 4: 360-366.

49. Xiao N, Callaway CW, Lipinski CA, Hicks SD, DeFranco DB. Geldanamycin provides posttreatment protection against glutamate-induced oxidative toxicity in a mouse hippocampal cell line. J Neurochem 1999; 72: 95-101. 